\title{
Case of HIV infection acquired by pre-mastication: first reported pediatric case in Chile
}

\begin{abstract}
Summary
In Chile, the incorporation of the protocol for the prevention of vertical transmission (VTPP) of human immunodeficiency virus (HIV) in pregnant women, has reduced HIV transmission in children to less than $2 \%$. Thus, currently the detection of HIV-infected children is uncommon. So, in front a case, compliance with the VTPP must be audited. If it was fully complied, other possible mechanism of HIV transmission should be reviewed. A case is presented in which, having discarded the common causes of contagion, HIV may have been transmitted through the consumption of pre-masked food by an HIV infected person, in this case the mother.
\end{abstract}

Keywords: HIV, vertical transmission, pre-mastication
Volume 6 Issue 2 - 2020

\author{
Elba Wu Hupat, Yenis Labraña Cornejo, Ana \\ María Alvarez, Julia Villarroel \\ West School of Medicine, University of Chile, Chile
}

Correspondence: Elba Wu Hupat, West School of Medicine, University of Chile, Miraflores 686, Appt. 50I, Santiago, Chile, Tel 56-2-26394I56,Email shuanwh@gmail.com

Received: February 15, 2020 | Published: March II, 2020
Abbreviations: HIV, human immunodeficiency virus; ISP, institute of public health; HIV VTPP, HIV vertical transmission prevention protocol

\section{Introduction}

Human immunodeficiency virus (HIV) infection in children is acquired in about $95 \%$ of cases vertically, during pregnancy, childbirth and breastfeeding, being most common in the last weeks of pregnancy-delivery. ${ }^{1}$ The incorporation in Chile of HIV vertical transmission prevention protocol (HIV VTPP) in pregnant women, including the use of anti-retroviral drugs in pregnancy, childbirth and in the newborn up to 6 weeks of life, the indication of C-section, premature membrane rupture of less than 4 hours and suspension of breastfeeding, have reduced the percentage of HIV transmission in children to less than $2 \%{ }^{2,3}$ Modes of transmission other than perinatal such as hemophilia, blood transfusions are currently very rare in Chile since HIV is tested in all blood banks and synthetic coagulation factors are used; sexual abuse is a mode of transmission that should always be sought. Even so, there are cases in which the risk factor cannot be identified or is not reported.

In Chile, with the unofficial incorporation of HIV VTPP in 1995 and by Ministry of Health (MINSAL) Norm HIV VTPP since 2005, the detection of an HIV-infected child is currently uncommon., Since 2005, with informed consent, the examination of HIV-specific antibodies in pregnant women has been offered, so confirmed cases quickly initiate the VTPP of this virus. Thus, when detecting an infected child, compliance with the entire VTPP process should be reviewed and if it was fully fulfilled, other possible routes of HIV transmission should be considered. ${ }^{2,3}$ A little-known mode of transmission of pathogens is pre-mastication. Food pre-mastication (chewing food before giving to a child) occurs mostly during weaning and is a common behavior that has been described in various parts of the world. The pre-mastication of solid foods seems to be common in some cultures and can be commonly used to support and supplement breastfeeding in areas where drinking water and formula are not easily available. $^{4}$

Various infectious agents such as bacteria, fungi and viruses can be transmitted through pre-mastication. Pre-mastication has been linked to the transmission of herpes 8 virus, Epstein Barr virus, Helicobacter pylori and HIV from infected mothers to their children. ${ }^{5-12}$ We present a pediatric case that having completed all stages of HIV VTPP, alerted us to look for other possible mechanisms of transmission and which the scientific literature has now incorporated in novel form.

\section{Clinical case}

Female patient, with a history of mother with HIV infection diagnosed at 20 weeks of gestation and that received VTPP with zidovudine, lamivudine and lopinavir/ritonavir from that moment. The delivery was by elective Caesarean and was indicated breastfeeding suspension, replacing it with artificial feeding. During the newborn period the diagnostic HIV infection study for children who are born with HIV infection was performed according to the Norm of the Institute of Public Health (ISP) in our country. The study shortly consists of detection of the viral genome (using PCR) at $1^{\text {st }}$ day of life, 15-30 days of birth and 3 months of age. The study was negative for HIV infection, being discharged from the polyclinic of Infectious Diseases at 18 months of life, with serology for HIV negative. At 2 years and 4 months he developed a picture of fever, intermittent diarrhea and general condition commitment. In the physical exam was found an extensive thrush and retro-auricular lymphadenopathies. Because of these manifestations and for the background of being the daughter of a mother with HIV infection, HIV serology was requested and was positive, result that was confirmed by the ISP. The child was classified in the clinical category B and in the immunological category $2 .{ }^{13}$ Was initiated in Antiretroviral Therapy with Abacavir, Lamivudine and Efavirenz.

The study of possible transmission mechanisms other than vertical was initiated. He had no history of transfusions, so the sexual pathway was raised as the most likely. She was evaluated by a psychologist and psychiatrist, those who did not find a sustainable history. It was also evaluated by two child gynecologists who ruled out signs of sexual abuse. A rectal endosonographic revision was performed that was normal, so sexual transmission could not be confirmed. Reinterrogating the mother and reviewing the recent scientific literature describing pre-mastication as a new form of HIV transmission, the mother confirmed practicing pre-chewing repeatedly.

\section{Discussion}

The case presented is consistent with the observation that HIV can be transmitted through the consumption of foods that have been pre- 
masked by an HIV-infected person., ${ }^{4,-12}$ Worldwide, transmission of HIV infection had not been associated with this practice until 2009. That year three cases of pediatric HIV infection were reported in children previously negative for HIV infection who received premasked food from an adult who was infected with the virus. In two cases, concurrent oral bleeding was described in the adult who premasticated the food. Phylogenetic analysis confirmed that two of the three children were infected through exposure to pre-masked food by an HIV-infected caregiver. They conducted in-depth research to rule out alternative modes of transmission (vertical transmission, breastfeeding, transfusions, sexual abuse). ${ }^{9}$ These cases provide conclusive evidence of the association of pre-mastication and HIV infection. This new route of transmission, which had not been previously reported, is a possible explanation of cases of "late" transmission of HIV in infants, hitherto attributed to breastfeeding. Pre-mastication has been reported as a form of transmission of HIV infection through blood into saliva that is mixed with chewed food.

The CDC conducted a cross-sectional survey at nine clinics in the U.S. between December 2009 and February 2010. It was found that of 154 primary caregivers, $31 \%$ of children exposed to HIV $\geq 6$ months received pre-masked food from a caregiver. Younger caregivers reported significantly higher rates of this practice compared to elderly, and black caregivers more often than caregivers of another race. Approximately $14 \%$ of caregivers in the U.S. reported pre-mastication. Biological mothers accounted for $79 \%$ of the pre-chewers. There were no differences in the incidence of pre-mastication based on the child's sex, the caregiver's country of origin, education level and income level. This practice began in children as young as one month old and was abandoned as belatedly as at the age of 36 months. The most frequently mentioned reasons for pre-mastication are: "the child wants some of the caregiver's food"( $64 \%)$, "the caregiver does not want the child chocking" $(62 \%)$ and "pre-mastication is done in my family" $(31 \%) .{ }^{11}$ The history of childhood exposure to pre-mastication could help identify HIV-infected caregivers who perform this practice. ${ }^{12}$

\section{Acknowledgments}

None.

\section{Conflicts of interest}

The author declares that he has no conflicts of interest.

\section{Funding}

None.

\section{References}

1. UNAIDS. UNAIDS Data. 2019.

2. CONASIDA: Ministry of Health, Chile. Norm for the Prevention of Vertical Transmission of HIV, August 2005, 2009 and 2013.

3. Institute of Public Health (ISP): Ministry of Health, Chile. Quarterly information, virologic diagnostic results of pediatric HIV infection.

4. Pelton GH, Zhang Y, Habicht JP. Pre-mastication: the second arm of infant and young child feeding for health and survival. Matern Child Nutr. 2010;6(1):4-18.

5. Wojcicki JM. Traditional behavior practices, the exchange of saliva and HHV-8 transmission in sub-Saharan African populations. Br J Cancer. 2003;89(0):2016-2017.

6. Mbulaiteye SM, Walters M, Engels EA, et al. High levels of EpsteinBarr virus DNA in saliva and peripheral blood from Ugandan motherchild pairs. J Infect Dis. 2006;193(3):422-426.

7. Taylor DN, Blaser MJ. The epidemiology of Helicobacter pylori infection. Epidemiol Rev. 1991;13:42-49.

8. González OA, Ebersole JL, Huang CB. Oral infectious diseases: a potential risk factor for HIV virus recrudescence? Oral Dis. 2009;15(5):313-327.

9. Gaur AH, Domínguez KL, Kalish ML, et al. Practice of feeding premasticated food to infants: a potential risk factor for HIV transmission. Pediatrics. 2009;124(2)658-666.

10. DiNubile MJ. Pre-mastication: a possible missing link? Clin Infect Dis. 2010;51:252-253.

11. Centers for Disease Control and Prevention (CDC). Pre-mastication of food by caregivers of HIV-exposed children-nine U.S. sites, 2009-2010. MMWR Morb Mortal Wkly Rep. 2011;60:273-275.

12. Hafeez S, Salami O, Alvarado M, et al. Infant feeding practice of premastication: an anonymous survey among human immunodeficiency virus-infected mothers. Arch Pediatr Adolesc Med. 2011;165(1):92-93.

13. CDC. Revised Classification system for human immunodeficiency virus infection in children less than 13 years of age. MMWR Morbid Mortal Wkly Rep. 1994;43(RR-12):1-10. 